\title{
Inpatient screening for albuminuria and retinopathy to predict long-term mortality in type 2 diabetic patients: a retrospective cohort study
}

\author{
Ya-Mei Hsieh ${ }^{1}$, Wen-Jane Lee ${ }^{2}$, Wayne H.-H. Sheu ${ }^{1,3,4}$, Yu-Hsuan Li ${ }^{1}$, Shih-Yi Lin ${ }^{5}$ and I.-Te Lee ${ }^{1,3,4^{*}}$
}

\begin{abstract}
Background: There is a high hospitalization rate for diabetic patients. Since retinopathy and albuminuria are both important manifestations of microvascular disease in diabetes, our aim was to investigate the effect of retinopathy and albuminuria on long-term mortality in type 2 diabetic inpatients through this observational cohort study.
\end{abstract}

Methods: Type 2 diabetic inpatients given a primary diagnosis of poor glucose control were consecutively enrolled during their hospitalization periods. Clinical information was collected through review of each patient's medical records, and mortality data were obtained from the national registry in Taiwan.

Results: A total of 761 type 2 diabetic inpatients were enrolled in the study with a median follow-up period of 6.6 years (interquartile range, 4.0-9.6 years). Patients in the Albuminuria(-)/Retinopathy(+), Albuminuria(+)/Retinopathy(-) and Albuminuria(+)/Retinopathy $(+)$ groups had significantly higher risks of all-cause mortality and cardiovascular mortality than those in the Albuminuria(-)/Retinopathy(-) group. However, among patients with albuminuria, there was no significant difference in cumulative mortality between those with and without retinopathy $(P=0.821)$. A decrease in the estimated glomerular filtration rate (eGFR), but not retinopathy, was an independent predictor of allcause mortality $(95 \% \mathrm{Cl} 0.647-0.893 ; \mathrm{P}<0.001)$ and cardiovascular mortality (95\% Cl 0.564-0.921; $\mathrm{P}=0.009)$ in type 2 diabetic inpatients with albuminuria.

Conclusions: Albuminuria in type 2 diabetic inpatients is a strong predictor of long-term mortality after discharge from the hospital. Retinopathy is an independent predictor of mortality in type 2 diabetic inpatients without albuminuria but not in those with albuminuria. A low eGFR is a better predictor of mortality than retinopathy in type 2 diabetic inpatients with albuminuria.

Keywords: Albuminuria, Inpatient, Mortality, Retinopathy

\section{Background}

Diabetes mellitus is a complex metabolic disorder, and poor blood glucose control is associated with sequential chronic microvascular complications [1]. Albuminuria not only indicates the presence of diabetic nephropathy but also predicts mortality [1-4]. Therefore, early

\footnotetext{
*Correspondence: itlee@vghtc.gov.tw

${ }^{1}$ Division of Endocrinology and Metabolism, Department of Internal

Medicine, Taichung Veterans General Hospital, No. 1650 Taiwan Boulevard,

Sect. 4, Taichung, 40705, Taiwan

Full list of author information is available at the end of the article
}

identification and prevention of albuminuria plays a vital part in the clinical management of diabetes [5].

In addition to nephropathy, retinopathy is another significant manifestation of microvascular disease in subjects with diabetes [1]. A high prevalence of diabetic retinopathy has been reported in diabetic patients with albuminuria [6, 7], and the mortality rate of diabetic patients diagnosed with both retinopathy and albuminuria is high [8]. Awareness of the presence of retinopathy is essential in all diabetic patients, not only in those with albuminuria $[1,9]$. However, the frequency of eye 
examinations and level of eye care are low in outpatient practice for diabetic patients [10].

Since there is a high hospitalization rate in subjects with diabetes [11], screening for albuminuria and retinopathy is practical for inpatients with diabetes. The long-term mortality rate of diabetic inpatients with albuminuria or retinopathy after discharge from the hospital has seldom been investigated. Therefore, we assessed the impact of albuminuria and retinopathy on long-term mortality in diabetic patients hospitalized due to poor blood glucose control.

\section{Methods}

\section{Subjects}

This study was conducted in the Endocrinology and Metabolism ward of Taichung Veterans General Hospital. Data collection was performed through the review of medical records of the diabetic patients hospitalized between August 1, 1996 and August 31, 2007. In general, all hospitalized type 2 diabetic patients, along with type 1 patients with a diabetic duration of more than 5 years, underwent urine collection and ophthalmology consultation for the evaluation of microvascular complications before being discharged. Patients were included in the study if (1) they were admitted due to a primary diagnosis of poor glucose control, (2) they had undergone ophthalmology consultation, and (3) urinary albumin excretion and serum creatinine had been assessed. Patients were excluded from analyses if (1) there were any inconsistent interpretations of eye assessments during hospitalization, (2) they had been hospitalized in critical condition, with a systolic blood pressure lower than $80 \mathrm{mmHg}$, or (3) they had died in the hospital. In the case of patients who had been hospitalized more than once during the study period, only the records of their last admission were analyzed.

\section{Assessments}

Mortality data up to December 31, 2011 were obtained from the Collaboration Center of Health Information Application, Department of Health, Executive Yuan, Taiwan. This study complied with the tenets of the Declaration of Helsinki, and the research protocol was approved by the Institutional Review Board of Taichung Veterans General Hospital.

Based on the standard procedure in our ward during this period, all fundoscopic data were reviewed by ophthalmologists. If any abnormal findings were discovered by their fundoscopic assessments, retinal angiography (CF-60UVi fundus camera, Canon, Japan) was performed to confirm a retinopathy diagnosis. Patients were excluded from the analysis if the interpretations were inconsistent between fundoscopy and angiography.
In the present study, we defined the presence of diabetic retinopathy including non-proliferative diabetic retinopathy (NPDR) and proliferative diabetic retinopathy (PDR) [12].

Laboratory analyses were performed according to the standard procedures of our ward. In brief, blood samples for biochemistry analyses were collected after an overnight fast. HbA1c was determined by cation-exchange high-pressure liquid chromatography (NGSP certificated; G8, TOSOH, Tokyo, Japan). Serum levels of total cholesterol, high-density lipoprotein (HDL) cholesterol and triglycerides were determined using enzymatic methods (Advia 1800, Siemens, New York, U.S.A.). Creatinine levels were determined using the Jaffé method (Advia 1800, Siemens, New York, U.S.A.), and urinary albumin levels were determined using the polyethylene glycol enhanced immuno turbidimetric method (Advia 1800, Siemens, New York, U.S.A.). The calculation of estimated glomerular filtration rate (eGFR) was applied by $186 \times$ [serum creatinine $(\mathrm{mg} / \mathrm{dL})]^{-1.154} \times$ age (year) $]^{-0.203}(\times 0.742$, if female) $\mathrm{mL} / \mathrm{min} / 1.73 \mathrm{~m}^{2}$ based on the modification of diet in renal disease (MDRD) study equation [2]. The urine albumin creatinine ratio (ACR) was determined by the ratio of urine albumin (in milligrams) to urine creatinine (in grams). Albuminuria was defined as an $\mathrm{ACR} \geq 300 \mathrm{mg} / \mathrm{g}[1,2]$. Hypertension was defined as a blood pressure higher than $130 / 80 \mathrm{mmHg}$ or a history of being prescribed anti-hypertensive medications.

\section{Statistical analysis}

Continuous data were presented as the mean \pm standard deviation (SD). One-way analysis of variance (ANOVA) was used to compare the differences among groups. Pairwise multiple comparisons were performed to determine the significance of differences between two groups after ANOVA had revealed any statistical significance. Chi square test was used to compare categorical variables across the groups. The overall significance of univariate survival analysis was detected through the use of the logrank test using Kaplan-Meier analysis. Multivariate Cox proportional hazards regression analyses were conducted to determine the hazard ratios. The statistical significance was set at $\mathrm{P}<0.05$. Statistical analysis was performed using SPSS 22.0 (IBM Corp., Armonk, NY, USA).

\section{Results}

A total of 855 admissions satisfied the inclusion criteria; however, 76 admissions were excluded, including 24 admissions with inconsistent retinopathy interpretation between fundoscopy and angiography, 4 admissions in which the patients died or underwent shock, and 48 admissions after which patients were repeatedly hospitalized during the study period. Furthermore, 17 patients 
were excluded due to having type 1 diabetes and one patient with diabetes due to chronic pancreatitis was also excluded in the enrollment. Therefore, a total of 761 diabetic inpatients were enrolled for analyses. Based on ACR, there were 207 (27.2\%) patients with albuminuria and $554(72.8 \%)$ without albuminuria. Based on fundoscopic examination, there were $330(43.4 \%)$ patients with retinopathy and 431 (56.6\%) without retinopathy among all study subjects. The prevalence of retinopathy was higher in patients with albuminuria than in those without $(66.7 \%$ vs. $34.7 \%, \mathrm{P}<0.001)$. Over a median period of 6.6 years (interquartile range, 4.0-9.6 years) after discharge from hospital, there were 409 (53.7\%) subjects who died during the follow-up period. Among the patients without albuminuria, the cumulative mortality rate of patients with retinopathy was significantly higher than that of patients without retinopathy $(58.3 \%$ vs. $39.2 \%$; $\mathrm{P}$ 0.001). However, among patients with albuminuria, the cumulative mortality rate was not significantly different between those with retinopathy and those without $(75.4 \%$ vs. $73.9 \%, \mathrm{P}=0.821$ ) (Fig. 1 ).

All study subjects were divided into four groups, including the patients with neither albuminuria nor retinopathy [Albuminuria(-)/Retinopathy $(-)$ ], patients

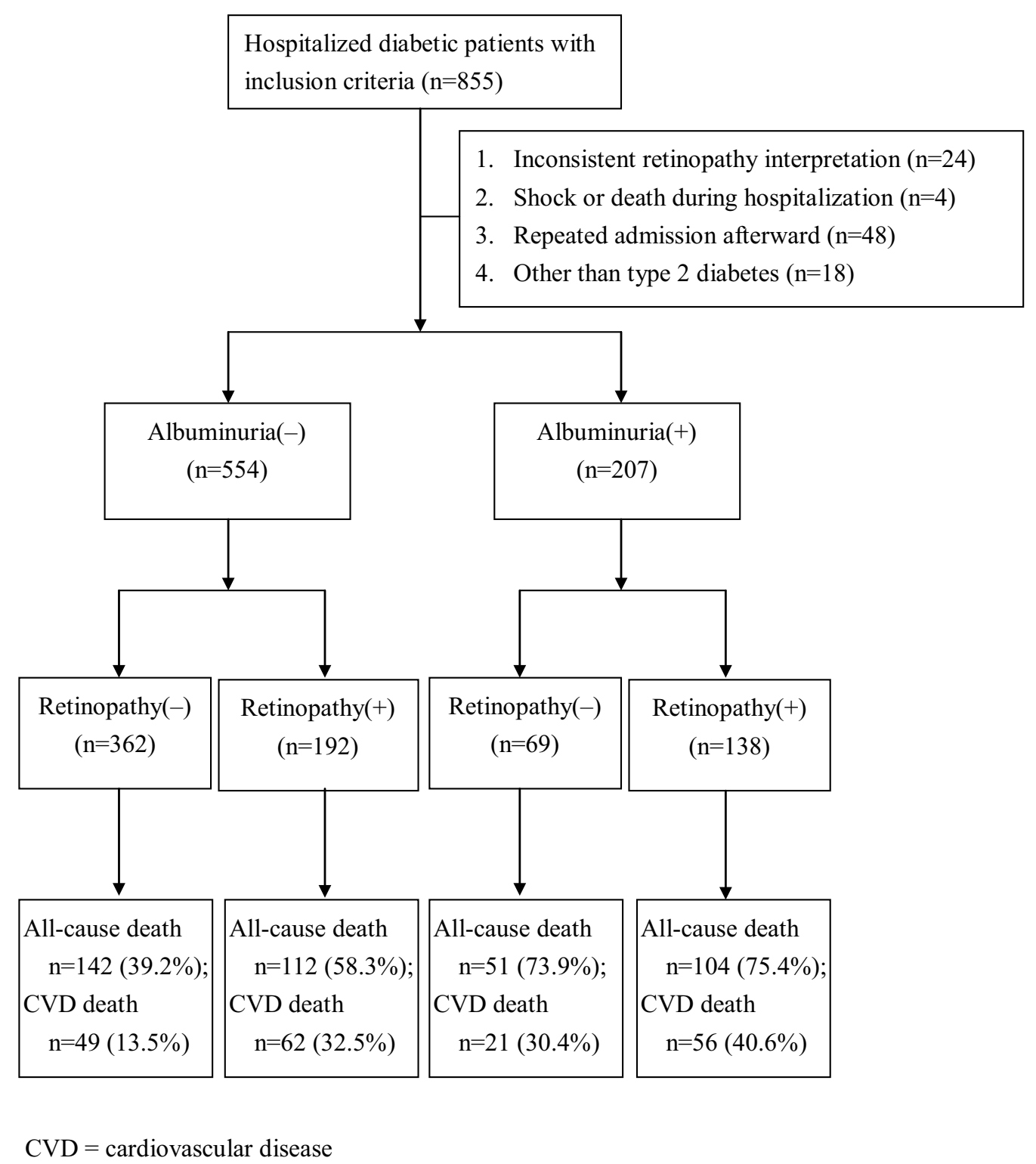

Fig. 1 The presence of albuminuria or retinopathy during the hospitalization, and the cumulative mortality rate after discharge from the hospital 
with retinopathy but no albuminuria [Albuminuria(-)/ Retinopathy $(+)]$, patients with albuminuria but no retinopathy [Albuminuria $(+) / \operatorname{Retinopathy}(-)]$, and patients with albuminuria and retinopathy [Albuminuria $(+) /$ Retinopathy $(+)]$. Table 1 shows the characteristics of these four groups. Figure 2 shows that the survival rate of the patients in the Albuminuria(-)/Retinopathy(-) group was highest among these four groups (log-rank test $\mathrm{P}<0.001$ ) using Kaplan-Meier analysis. Among the patients without albuminuria, the incidence of mortality in patients with retinopathy was significantly higher than that of patients without retinopathy $(8.4$ vs. 5.3 events/100 person-year; $\mathrm{P}<0.001$ ). However, among patients with albuminuria, the incidence of mortality was not significantly different between those with retinopathy and those without (11.9 vs. 14.2 events/100 person-year; $\mathrm{P}=0.310$ ).
During the follow-up period, Cox regression analysis showed that patients in the Albuminuria(-)/Retinopathy $(-)$ group had the lowest risk for all-cause mortality and cardiovascular mortality. Patients in the Albuminu$\mathrm{ria}(-) /$ Retinopathy $(+)$ group had significantly higher all-cause mortality (hazard ratio $[\mathrm{HR}]=1.524,95 \%$ confidence interval $[\mathrm{CI}] 1.138-2.041)$ and cardiovascular mortality $(\mathrm{HR}=2.434,95 \% \mathrm{CI} 1.562-3.794)$ than patients in the Albuminuria(-)/Retinopathy $(-)$ group. Patients in the Albuminuria(+)/Retinopathy(-) group had significantly higher all-cause mortality $(\mathrm{HR}=2.551,95 \% \mathrm{CI}$ 1.771-3.676) and cardiovascular mortality $(\mathrm{HR}=2.762$, 95\% CI 1.551-4.917), and patients in the Albuminu$\mathrm{ria}(+) /$ Retinopathy $(+)$ group also had significantly higher all-cause mortality $(\mathrm{HR}=2.200,95 \%$ CI $1.613-$ $3.001)$ and cardiovascular mortality $(\mathrm{HR}=3.327,95 \%$ CI 2.080-5.321) than patients in the Albuminuria(-)/

Table 1 The clinical data of patients according to the presence or absence of albuminuria and retinopathy

\begin{tabular}{|c|c|c|c|c|c|}
\hline & $\begin{array}{l}\text { Albuminuria(-)/ } \\
\text { Retinopathy(-) } \\
(\mathrm{n}=362)\end{array}$ & $\begin{array}{l}\text { Albuminuria(-)/ } \\
\text { Retinopathy(+) } \\
(n=192)\end{array}$ & $\begin{array}{l}\text { Albuminuria(+) } \\
\text { Retinopathy(-) } \\
(\mathrm{n}=69)\end{array}$ & $\begin{array}{l}\text { Albuminuria(+) } \\
\text { Retinopathy }(+) \\
(n=138)\end{array}$ & $\mathbf{P}$ \\
\hline Age (years) & $61 \pm 15$ & $66 \pm 12$ & $68 \pm 12$ & $65 \pm 11$ & $<0.001$ \\
\hline Male, n (\%) & $224(61.9 \%)$ & $98(51.0 \%)$ & $37(53.6 \%)$ & $70(50.7 \%)$ & 0.034 \\
\hline $\mathrm{BMI}\left(\mathrm{kg} / \mathrm{m}^{2}\right)$ & $24.1 \pm 4.1$ & $23.6 \pm 4.2$ & $24.4 \pm 3.8$ & $24.1 \pm 4.0$ & 0.528 \\
\hline Systolic blood pressure $(\mathrm{mmHg})$ & $127 \pm 16$ & $130 \pm 15$ & $137 \pm 16$ & $142 \pm 15$ & $<0.001$ \\
\hline Diastolic blood pressure $(\mathrm{mmHg})$ & $75 \pm 11$ & $74 \pm 10$ & $77 \pm 10$ & $79 \pm 10$ & $<0.001$ \\
\hline Diabetes duration (years) & $6.9 \pm 6.7$ & $12.3 \pm 7.8$ & $10.5 \pm 8.2$ & $12.3 \pm 7.3$ & $<0.001$ \\
\hline Current smoker, n (\%) & $109(30.1 \%)$ & $48(25.0 \%)$ & $21(30.4 \%)$ & $35(25.4 \%)$ & 0.506 \\
\hline White blood cell count $\left(10^{6} / \mathrm{L}\right)$ & $8045 \pm 3199$ & $8193 \pm 3565$ & $8850 \pm 3988$ & $8389 \pm 2917$ & 0.412 \\
\hline $\mathrm{HbA1c}(\%)$ & $11.1 \pm 3.1$ & $10.3 \pm 2.7$ & $10.7 \pm 2.9$ & $9.3 \pm 2.4$ & $<0.001$ \\
\hline Total cholesterol (mmol/L) & $4.8 \pm 1.5$ & $4.7 \pm 1.3$ & $5.0 \pm 1.5$ & $5.4 \pm 1.8$ & $<0.001$ \\
\hline Triglyceride (mmol/L) & $2.2 \pm 2.8$ & $1.7 \pm 1.2$ & $2.1 \pm 1.4$ & $2.3 \pm 2.6$ & 0.044 \\
\hline HDL cholesterol (mmol/L) & $1.0 \pm 0.3$ & $1.1 \pm 0.4$ & $1.0 \pm 0.4$ & $1.0 \pm 0.4$ & 0.127 \\
\hline $\mathrm{eGFR}\left(\mathrm{mL} / \mathrm{min} / 1.73 \mathrm{~m}^{2}\right)$ & $73 \pm 33$ & $68 \pm 29$ & $52 \pm 28$ & $53 \pm 30$ & $<0.001$ \\
\hline Albumin to creatinine ratio $(\mathrm{mg} / \mathrm{g})$ & $54 \pm 64$ & $98 \pm 83$ & $1226 \pm 1285$ & $2825 \pm 5043$ & $<0.001$ \\
\hline Hypertension, n (\%) & $246(68.0 \%)$ & $145(75.5 \%)$ & $57(82.6 \%)$ & $125(90.6 \%)$ & $<0.001$ \\
\hline Antihypertensive agents, n (\%) & $165(45.6 \%)$ & $110(57.3 \%)$ & $40(58.0 \%)$ & $81(58.7 \%)$ & 0.009 \\
\hline ACE inhibitor or ARB, n (\%) & $110(30.4 \%)$ & $68(35.4 \%)$ & $25(36.2 \%)$ & $61(44.2 \%)$ & 0.035 \\
\hline a-Blocker, n (\%) & $47(13.0 \%)$ & $26(13.5 \%)$ & $11(15.9 \%)$ & $13(9.4 \%)$ & 0.545 \\
\hline$\beta$-Blocker, n (\%) & $20(5.5 \%)$ & $17(8.9 \%)$ & $5(7.2 \%)$ & $10(7.2 \%)$ & 0.520 \\
\hline Calcium channel blocker, n (\%) & $66(18.2 \%)$ & $51(26.6 \%)$ & $27(39.1 \%)$ & $39(28.3 \%)$ & $<0.001$ \\
\hline Diuretics, n (\%) & $20(5.5 \%)$ & $17(8.9 \%)$ & $9(13.0 \%)$ & $22(15.9 \%)$ & 0.002 \\
\hline Oral antihyperglycemic drugs, n (\%) & $158(43.6 \%)$ & $75(39.1 \%)$ & 31 (44.9\%) & $37(26.8 \%)$ & 0.005 \\
\hline Insulin secretagogues, n (\%) & $139(38.4 \%)$ & $64(33.3 \%)$ & $29(42.0 \%)$ & $34(24.6 \%)$ & 0.018 \\
\hline Metformin, n (\%) & $84(23.2 \%)$ & $48(25.0 \%)$ & $16(23.2 \%)$ & $20(14.5 \%)$ & 0.117 \\
\hline Thiazolidinediones, n (\%) & $7(1.9 \%)$ & $4(2.1 \%)$ & $0(0.0 \%)$ & $0(0.0 \%)$ & 0.242 \\
\hline a-Glucosidase inhibitor, n (\%) & $6(1.7 \%)$ & $4(2.1 \%)$ & $1(1.4 \%)$ & $0(0.0 \%)$ & 0.443 \\
\hline Insulin therapy, n (\%) & $224(61.9 \%)$ & $99(51.6 \%)$ & $35(50.7 \%)$ & $42(30.4 \%)$ & $<0.001$ \\
\hline Statins, n (\%) & $39(10.8 \%)$ & $18(9.4 \%)$ & $4(5.8 \%)$ & $15(10.9 \%)$ & 0.616 \\
\hline
\end{tabular}

Statistical significance $(P<0.05)$ is indicated in italics

$A C E$ angiotensin-converting enzyme, $A R B$ angiotensin II receptor blocker, $B M I$ body mass index, eGFR estimated glomerular filtration rate, $H b A 1 c$ glycated hemoglobin, $H D L$ high-density lipoprotein 


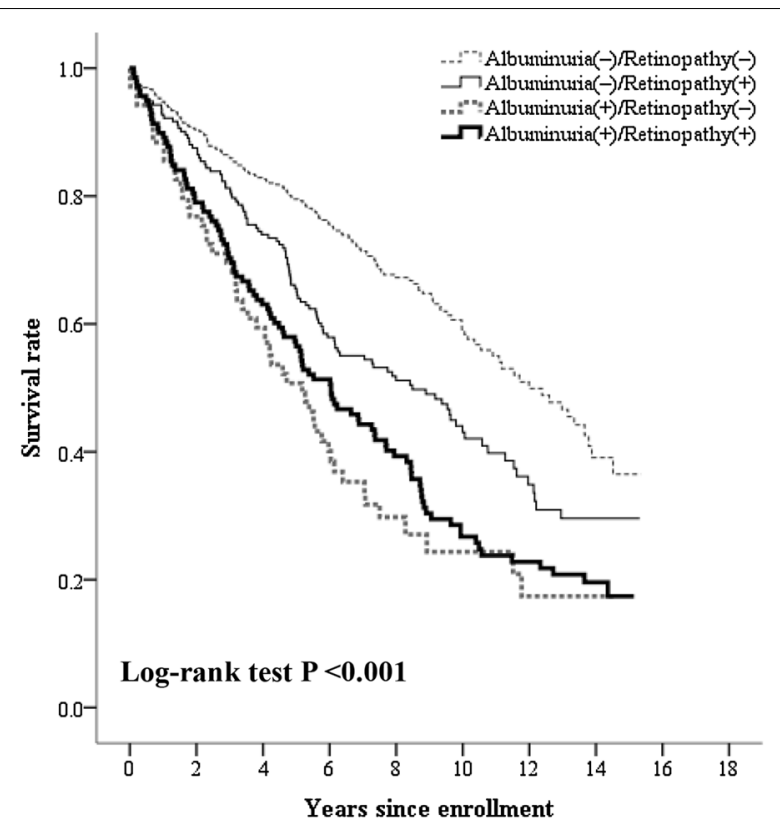

Fig. 2 Kaplan-Meier curves showing survival rates grouped by the presence or absence of retinopathy and albuminuria

Retinopathy(-) group. These conclusions were made after data were adjusted for age, gender, body mass index, systolic blood pressure, current smoker, diabetes duration, HbA1c, chronic kidney disease, total cholesterol, triglycerides, insulin treatment, and angiotensin-converting enzyme inhibitor/angiotensin II receptor blocker treatment (Table 2).

In the patients with albuminuria, Table $3 \mathrm{~A}$ shows that presence of retinopathy was not a significant predictor for all-cause mortality either in univariate analyses or in multivariate analyses after being adjusted for albuminuria (both $P>0.05$ ). However, eGFR was a significant predictor for all-cause mortality both in univariate analyses and in multivariate analyses (both $\mathrm{P}<0.001$ ). Similarly, in the patients with albuminuria, Table $3 \mathrm{~B}$ shows that the presence of retinopathy was not a significant predictor for cardiovascular mortality either in univariate analyses or in multivariate analyses after being adjusted for albuminuria (both $P>0.05$ ). ACR and eGFR were significant predictors for cardiovascular mortality both in univariate analyses $(P=0.041$ for ACR and 0.013 for eGFR, respectively) and in multivariate analyses $(\mathrm{P}=0.039$ for ACR, and 0.009 for eGFR, respectively).

\section{Discussion}

Diabetic retinopathy is associated with systemic vascular inflammation [13, 14], and it increases the risk of mortality $[9,15,16]$. Despite a high mortality rate in the patients with both retinopathy and macroalbuminuria, the presence of retinopathy did not significantly increase the risk of all-cause or cardiovascular mortality in the subgroup of normoalbuminuria in a Chinese diabetic population [8]. Similarly, in an analysis based on the data from a National Health and Nutrition Examination Survey (NHANES), it was found that presence of retinopathy did not significantly predict total mortality in the subgroup of the patients without albuminuria [17]. However, based on fundoscopic screens during hospitalization, our results showed that retinopathy increased the risk of long-term mortality by $52.4 \%$ in type 2 diabetic inpatients without albuminuria. Since retinopathy is not rare in diabetic patients without albuminuria $[18,19]$, routine ophthalmology consultation is strongly recommended for diabetic inpatients, especially for those without albuminuria [20].

Previous research has shown that albuminuria is a predictor of mortality in Asian as well as Caucasian diabetic patients [21, 22]. Recently, Aragón-Sánchez et al. [23] reported that albuminuria was associated with in-hospital deaths of diabetic patients with foot complications. In the present cohort, over a median period of 6.6 years after discharge from hospital, the type 2 diabetic patients with albuminuria had a higher risk of all-cause and cardiovascular mortality than those without albuminuria detected during hospitalization. This result outlines the importance for assessment of albuminuria in hospitalized type 2 diabetic patients.

There is a high prevalence of retinopathy among diabetic patients with albuminuria [24]. Therefore, the impact of retinopathy on mortality could be attributed to its interaction with albuminuria $[8,25]$. It has been reported that albuminuria is a stronger predictor of mortality than retinopathy and that retinopathy is not an independent predictor of mortality after adjusting for the presence of albuminuria in the diabetic population [25]. Our findings provide evidence that retinopathy is a significant predictor of mortality in type 2 diabetic inpatients without albuminuria but not in those with albuminuria. It is noteworthy that a low eGFR, but not the presence of retinopathy, was an independent predictor of mortality in type 2 diabetic inpatients with albuminuria in the present study. Bello et al. also showed that retinopathy could not predict all-cause mortality in diabetic patients with chronic kidney disease [26]. Since eGFR and albuminuria showed a synergistic effect on mortality [27, 28], the calculation of eGFR is important for type 2 diabetic patients with albuminuria during hospitalization.

The mortality rate of the diabetic population is 1.63fold higher in comparison to the general population based on the data from the National Register of Deaths [29]. Furthermore, the mortality rate of hospitalized diabetic patients was 2.98 -fold higher than that of Taiwan's 


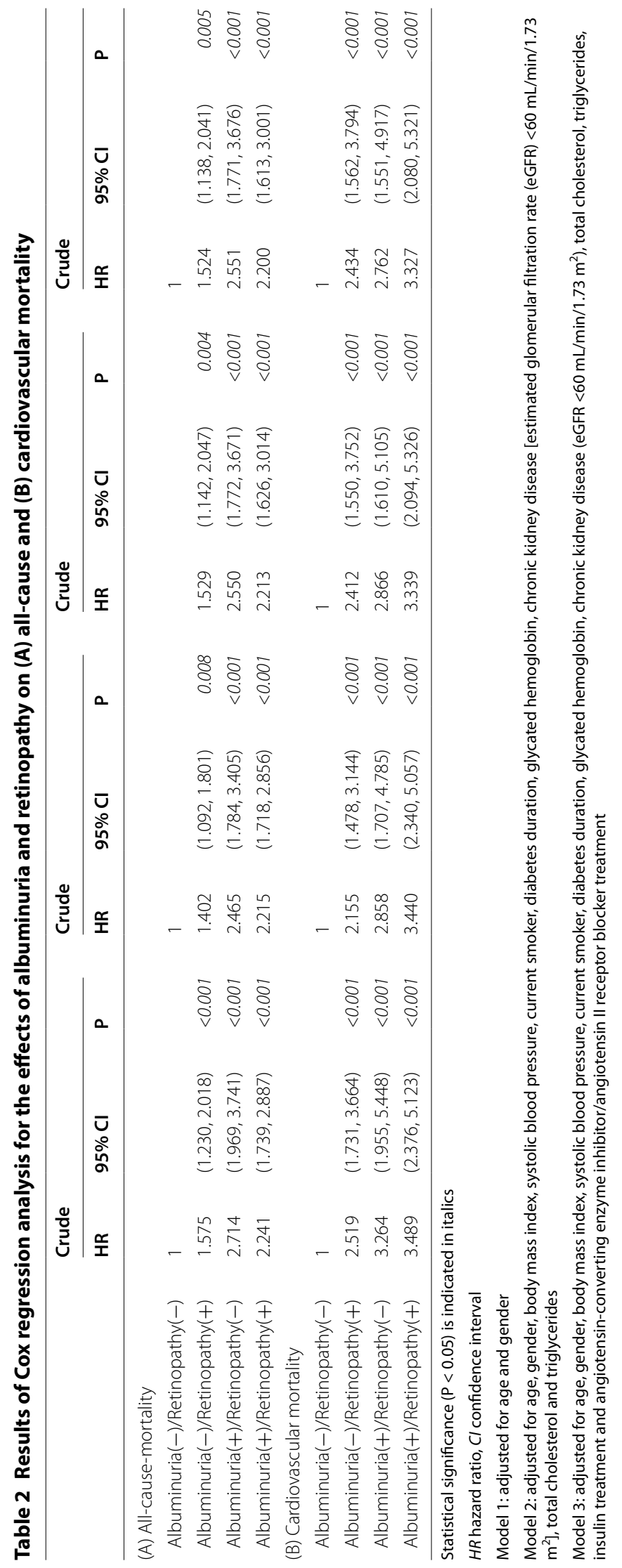


Table 3 Results of Cox regression analysis for the effects of risk factors on (A) all-cause and (B) cardiovascular mortality in patients with albuminuria

\begin{tabular}{|c|c|c|c|c|c|c|c|c|c|}
\hline & \multirow{2}{*}{\multicolumn{3}{|c|}{$\begin{array}{l}\text { Univariate model } \\
\text { Crude }\end{array}$}} & \multicolumn{6}{|c|}{ Multivariate model $^{\mathrm{a}}$} \\
\hline & & & & \multicolumn{3}{|c|}{ Model 1} & \multicolumn{3}{|c|}{ Model 2} \\
\hline & HR & $95 \% \mathrm{Cl}$ & $\mathbf{P}$ & HR & $95 \% \mathrm{Cl}$ & $\mathbf{P}$ & HR & $95 \% \mathrm{Cl}$ & $\mathbf{P}$ \\
\hline \multicolumn{10}{|l|}{ (A) All-cause mortality } \\
\hline Retinopathy (yes/no) & 0.840 & $(0.599,1.177)$ & 0.310 & 0.850 & $(0.595,1.213)$ & 0.370 & 0.672 & $(0.409,1.104)$ & 0.117 \\
\hline Urine albumin to creatinine ratio ${ }^{b}$ & 1.167 & $(0.820,1.662)$ & 0.390 & 1.359 & $(0.950,1.945)$ & 0.094 & 1.062 & $(0.664,1.699)$ & 0.801 \\
\hline eGFR (every 15 mL/min/1.73 m²) & 0.843 & $(0.769,0.924)$ & $<0.001$ & & & & 0.760 & $(0.647,0.893)$ & $<0.001$ \\
\hline Current smoker (yes/no) & 1.127 & $(0.790,1.606)$ & 0.509 & & & & 0.890 & $(0.517,1.532)$ & 0.675 \\
\hline Diabetes duration (every 1 year) & 1.012 & $(0.991,1.033)$ & 0.271 & & & & 0.996 & $(0.967,1.025)$ & 0.781 \\
\hline BMI (every 1 kg/m²) & 0.980 & $(0.929,1.033)$ & 0.453 & & & & 0.981 & $(0.920,1.045)$ & 0.551 \\
\hline Systolic blood pressure (every $10 \mathrm{mmHg}$ ) & 1.008 & $(0.906,1.121)$ & 0.886 & & & & 0.958 & $(0.826,1.110)$ & 0.567 \\
\hline HbA1c (every 1\%) & 1.004 & $(0.939,1.074)$ & 0.895 & & & & 1.024 & $(0.933,1.122)$ & 0.621 \\
\hline Total cholesterol (every 1 mmol/L) & 0.949 & $(0.857,1.050)$ & 0.313 & & & & 1.073 & $(0.920,1.251)$ & 0.368 \\
\hline Triglycerides (every 1 mmol/L) & 0.985 & $(0.917,1.058)$ & 0.677 & & & & 0.987 & $(0.908,1.073)$ & 0.762 \\
\hline \multicolumn{10}{|l|}{ (B) Cardiovascular mortality } \\
\hline Retinopathy (yes/no) & 1.111 & $(0.671,1.840)$ & 0.683 & 1.074 & $(0.632,1.825)$ & 0.793 & 0.937 & $(0.433,2.028)$ & 0.868 \\
\hline Albumin to creatinine ratio ${ }^{b}$ & 1.636 & $(1.021,2.623)$ & 0.041 & 1.839 & $(1.141,2.965)$ & 0.012 & 1.965 & $(1.035,3.730)$ & 0.039 \\
\hline eGFR (every 15 mL/min/1.73 m²) & 0.849 & $(0.745,0.966)$ & 0.013 & & & & 0.721 & $(0.564,0.921)$ & 0.009 \\
\hline Current smoker (yes/no) & 1.222 & $(0.745,2.004)$ & 0.428 & & & & 1.039 & $(0.429,2.518)$ & 0.932 \\
\hline Diabetes duration (every 1 year) & 1.029 & $(1.000,1.059)$ & 0.049 & & & & 1.011 & $(0.969,1.055)$ & 0.610 \\
\hline BMI (every 1 kg/m²) & 1.003 & $(0.931,1.080)$ & 0.942 & & & & 1.010 & $(0.918,1.110)$ & 0.843 \\
\hline Systolic BP (every 10 mmHg) & 1.041 & $(0.876,1.237)$ & 0.645 & & & & 0.974 & $(0.776,1.224)$ & 0.824 \\
\hline HbA1c (every 1\%) & 1.021 & $(0.921,1.133)$ & 0.689 & & & & 1.049 & $(0.908,1.212)$ & 0.515 \\
\hline Total cholesterol (every 1 mmol/L) & 0.861 & $(0.693,1.071)$ & 0.179 & & & & 0.952 & $(0.744,1.219)$ & 0.697 \\
\hline Triglycerides (every 1 mmol/L) & 0.946 & $(0.748,1.197)$ & 0.645 & & & & 1.003 & $(0.883,1.139)$ & 0.967 \\
\hline
\end{tabular}

Statistical significance $(P<0.05)$ is indicated in italics

$B M I$ body mass index, eGFR estimated glomerular filtration rate, $H b A 1 c$ glycated hemoglobin, $C l$ confidence interval, $H R$ hazard ratio, $B P$ blood pressure

a After adjusted for age and gender

b Because of the skewed distribution, the albumin to creatinine ratio was logarithm-transformed (log) for the analyses

general population [30]. Although the mortality rate of diabetic patients has decreased in recent decades [31], there was still a high cumulative mortality rate (53.7\%) in type 2 diabetic inpatients admitted due to poor blood glucose control in the present study. Particularly in the type 2 diabetic inpatients with albuminuria, the mortality rate reached as high as $74.9 \%$ following a median time of 6.6 years upon discharge. Lipska et al. also found a high mortality rate of approximately $17 \%$ in diabetic inpatients within one year of discharge from the hospital [11]. The predictive factors of mortality for diabetic inpatients are emergent, and require further investigation.

Screening for retinopathy and nephropathy in diabetic patients is mostly done in the outpatient department. In view of the high mortality rate in diabetic inpatients admitted for poor glucose control, it is essential to routinely assess these predictors of mortality during hospitalization. However, evidence of the value of screening for retinopathy and albuminuria in hospitalized diabetic patients has not previously been adequately presented. Although various causes may bring about hyperglycemia in hospitalized type 2 diabetic patients, our findings suggest that routine screening for retinopathy and urine albumin excretion can be helpful in predicting mortality after discharge. Furthermore, as noted in a previous report, approximately $38 \%$ of hospitalized patients have diabetes, and more than $30 \%$ of those are newly diagnosed cases [32]. Therefore, inpatient assessment of retinopathy and nephropathy is also useful in the early detection of diabetes-associated complications [33].

There were some limitations in the present study. First, there were daily variations in urinary albumin excretion, so one should not rely on a single measurement [34]. However, we used only the ACR that was detected once during the hospitalization in this study. Second, we did not take into consideration any treatment that the patients received after discharge. Third, our cohort included only type 2 diabetic patients admitted with a primary diagnosis 
of poor blood glucose control, meaning our findings cannot be generalized for all diabetic patients.

\section{Conclusions}

The presence of albuminuria is an important predictor of long-term mortality in type 2 diabetic inpatients admitted due to poor blood glucose control. In type 2 diabetic inpatients without albuminuria, the presence of retinopathy is significantly associated with a higher long-term mortality. In type 2 diabetic inpatients with albuminuria, a low eGFR, but not the presence of retinopathy, is significantly associated with higher long-term mortality. In view of the increased risk of mortality after discharge, it is important to screen all hospitalized type 2 diabetic patients for nephropathy and retinopathy.

\section{Abbreviations}

ACE: angiotensin-converting enzyme; ACR: albumin creatinine ratio; ANOVA: analysis of variance; ARB: angiotensin II receptor blocker; BMI: body mass index; Cl: confidence interval; eGFR: estimated glomerular filtration rate; HbA1c: glycated hemoglobin; HDL: high-density lipoprotein; HR: hazard ratio; MDRD: modification of diet in renal disease; NPDR: non-proliferative diabetic retinopathy; PDR: proliferative diabetic retinopathy; SD: standard deviation.
\end{abstract}

\section{Authors' contributions}

IL contributed to the study design. WL, WHS, SL and IL participated in the data collection. $Y L$ and IL participated in the analysis and interpretation of the data. YH drafted the manuscript. IL had full access to the data in the study. IL is the guarantor. All the authors performed a critical revision of the manuscript for important intellectual content. All authors read and approved the final manuscript.

\begin{abstract}
Author details
${ }^{1}$ Division of Endocrinology and Metabolism, Department of Internal Medicine, Taichung Veterans General Hospital, No. 1650 Taiwan Boulevard, Sect. 4, Taichung, 40705, Taiwan. ${ }^{2}$ Department of Medical Research, Taichung Veterans General Hospital, Taichung 40705, Taiwan. ${ }^{3}$ School of Medicine, National Yang-Ming University, Taipei 11221, Taiwan. ${ }^{4}$ School of Medicine, Chung Shan Medical University, Taichung 40201, Taiwan. ${ }^{5}$ Center for Geriatrics and Gerontology, Taichung Veterans General Hospital, Taichung 40705, Taiwan.
\end{abstract}

\section{Acknowledgements}

Mortality data were provided by the Collaboration Center of Health Information Application, Ministry of Health and Welfare, Executive Yuan. The statistical analysis was performed by the Biostatistics Task Force of Taichung Veterans General Hospital, Taichung, Taiwan.

\section{Competing interests}

The authors declare that they have no competing interests.

\section{Availability of data and materials}

The datasets during and/or analyzed during the current study are available from the corresponding author on reasonable request.

\section{Ethics approval and consent to participate}

This study complied with the tenets of the Declaration of Helsinki. The Institutional Review Board of Taichung Veterans General Hospital approved this protocol and waived the requirement for informed consent (reference number: (E11279A).

\section{Funding}

This work was supported by Taichung Veterans General Hospital, Taichung, Taiwan (Grant Numbers TCVGH-1063502C, TCVGH-1063501E) and the Ministry of Science and Technology, Taiwan (MOST 105-2314-B-075A-003). The funders had no role in the decision to submit the manuscript for publication.

\section{Publisher's Note}

Springer Nature remains neutral with regard to jurisdictional claims in published maps and institutional affiliations.

Received: 23 February 2017 Accepted: 26 April 2017

Published online: 03 May 2017

\section{References}

1. American Diabetes Association. Standards of medical care in diabetes-2016: summary of revisions. Diabetes Care. 2016;39(Suppl 1):S4-5.

2. Inker LA, Astor BC, Fox CH, Isakova T, Lash JP, Peralta CA, et al. KDOQI US commentary on the $2012 \mathrm{KDIGO}$ clinical practice guideline for the evaluation and management of CKD. Am J Kidney Dis. 2014;63:713-35.

3. Gall MA, Borch-Johnsen K, Hougaard P, Nielsen FS, Parving HH. Albuminuria and poor glycemic control predict mortality in NIDDM. Diabetes. 1995:44:1303-9.

4. Gall MA, Hougaard P, Borch-Johnsen K, Parving HH. Risk factors for development of incipient and overt diabetic nephropathy in patients with non-insulin dependent diabetes mellitus: prospective, observational study. BMJ. 1997;314:783-8.

5. Ozyilmaz A, de Jong PE, Gansevoort RT. Screening for chronic kidney disease can be of help to prevent atherosclerotic end-organ damage. Nephrol Dial Transplant. 2012;27:4046-52.

6. Rani PK, Raman R, Gupta A, Pal SS, Kulothungan V, Sharma T. Albuminuria and diabetic retinopathy in type 2 diabetes mellitus sankara nethralaya diabetic retinopathy epidemiology and molecular genetic study (SNDREAMS, report 12). Diabetol Metab Syndr. 2011;3:9.

7. Constable IJ, Knuiman MW, Welborn TA, Cooper RL, Stanton KM, McCann VJ, et al. Assessing the risk of diabetic retinopathy. Am J Ophthalmol. 1984;97:53-61.

8. Tong PC, Kong AP, So WY, Yang X, Ng MC, Ho CS, et al. Interactive effect of retinopathy and macroalbuminuria on all-cause mortality, cardiovascular and renal end points in Chinese patients with Type 2 diabetes mellitus. Diabet Med. 2007;24:741-6.

9. Kramer CK, Rodrigues TC, Canani LH, Gross JL, Azevedo MJ. Diabetic retinopathy predicts all-cause mortality and cardiovascular events in both type 1 and 2 diabetes: meta-analysis of observational studies. Diabetes Care. 2011;34:1238-44.

10. Maclennan PA, McGwin G Jr, Heckemeyer C, Lolley VR, Hullett S, Saaddine $J$, et al. Eye care use among a high-risk diabetic population seen in a public hospital's clinics. JAMA Ophthalmol. 2014;132:162-7.

11. Lipska KJ, Ross JS, Wang Y, Inzucchi SE, Minges K, Karter AJ, et al. National trends in US hospital admissions for hyperglycemia and hypoglycemia among medicare beneficiaries, 1999 to 2011. JAMA Intern Med. 2014;174:1116-24.

12. Wilkinson CP, Ferris FL 3rd, Klein RE, Lee PP, Agardh CD, Davis M, et al. Proposed international clinical diabetic retinopathy and diabetic macular edema disease severity scales. Ophthalmology. 2003;110:1677-82.

13. Lv Z, Li Y, Wu Y, Qu Y. Association of ICAM-1 and HMGA1 gene variants with retinopathy in type 2 diabetes mellitus among Chinese individuals. Curr Eye Res. 2016;41:1118-22.

14. Semeraro F, Cancarini A, dell'Omo R, Rezzola S, Romano MR, Costagliola C. Diabetic retinopathy: vascular and inflammatory disease. J Diabetes Res. 2015;2015:582060.

15. Davis MD, Hiller R, Magli YL, Podgor MJ, Ederer F, Harris WA, et al. Prognosis for life in patients with diabetes: relation to severity of retinopathy. Trans Am Ophthalmol Soc. 1979;77:144-70.

16. van Hecke MV, Dekker JM, Stehouwer CD, Polak BC, Fuller JH, Sjolie AK, et al. Diabetic retinopathy is associated with mortality and cardiovascular disease incidence: the EURODIAB prospective complications study. Diabetes Care. 2005;28:1383-9.

17. Ricardo AC, Grunwald JE, Parvathaneni S, Goodin S, Ching A, Lash JP. Retinopathy and CKD as predictors of all-cause and cardiovascular 
mortality: National Health and Nutrition Examination Survey (NHANES) 1988-1994. Am J Kidney Dis. 2014;64:198-203.

18. Karoli R, Fatima J, Shukla V, Garg P, Ali A. Predictors of diabetic retinopathy in patients with type 2 diabetes who have normoalbuminuria. Ann Med Health Sci Res. 2013;3:536-40.

19. Ra H, Yoo JH, Ban WH, Song HC, Lee SS, Kim SR, et al. Predictors for diabetic retinopathy in normoalbuminuric people with type 2 diabetes mellitus. Diabetol Metab Syndr. 2012;4:29.

20. Tajunisah I, Azida J, Zurina ZA, Reddy SC. Ophthalmology inpatient consultation: does it make a difference to inpatient management? Med J Malaysia. 2009:64:130-3

21. Wada T, Haneda M, Furuichi K, Babazono T, Yokoyama H, Iseki K, et al. Clinical impact of albuminuria and glomerular filtration rate on renal and cardiovascular events, and all-cause mortality in Japanese patients with type 2 diabetes. Clin Exp Nephrol. 2014;18:613-20.

22. Svensson MK, Cederholm J, Eliasson B, Zethelius B, Gudbjornsdottir S, Swedish National Diabetes R. Albuminuria and renal function as predictors of cardiovascular events and mortality in a general population of patients with type 2 diabetes: a nationwide observational study from the Swedish National Diabetes Register. Diab Vasc Dis Res. 2013;10:520-9.

23. Aragon-Sanchez J, Lazaro-Martinez JL, Garcia-Alvarez Y, Morales EG, Hernandez-Herrero MJ. Albuminuria is a predictive factor of in-hospital mortality in patients with diabetes admitted for foot disease. Diabetes Res Clin Pract. 2014;104:e23-5.

24. Rodriguez-Poncelas A, Mundet-Tuduri X, Miravet-Jimenez S, Casellas A, Barrot-De la Puente JF, Franch-Nadal J, et al. Chronic kidney disease and diabetic retinopathy in patients with type 2 diabetes. PLoS ONE. 2016;11:e0149448.

25. Torffvit O, Lovestam-Adrian M, Agardh E, Agardh CD. Nephropathy, but not retinopathy, is associated with the development of heart disease in type 1 diabetes: a 12-year observation study of 462 patients. Diabet Med. 2005;22:723-9.
26. Bello NA, Pfeffer MA, Skali H, McGill JB, Rossert J, Olson KA, et al. Retinopathy and clinical outcomes in patients with type 2 diabetes mellitus, chronic kidney disease, and anemia. BMJ Open Diabetes Res Care. 2014;2:e000011.

27. Toyama T, Furuichi K, Ninomiya T, Shimizu M, Hara A, Iwata Y, et al. The impacts of albuminuria and low eGFR on the risk of cardiovascular death, all-cause mortality, and renal events in diabetic patients: meta-analysis. PLoS ONE. 2013;8:e71810

28. Lee IT, Sheu WH, Lin SY. The impact of creatinine clearance rate, daily urinary albumin, and their joint effect on predicting death in diabetic inpatients after discharge: an observational study. Medicine (Baltimore). 2016;95:e2804

29. Tseng CH. Mortality and causes of death in a national sample of diabetic patients in Taiwan. Diabetes Care. 2004:27:1605-9.

30. Wu MC, Lee WJ, Tschen SM, Lin SY, Lee IT, Jeng CY, et al. Predictors of mortality in hospitalized diabetic patients: a 7-year prospective study. Diabetes Res Clin Pract. 2008;80:449-54.

31. Li HY, Jiang YD, Chang CH, Chung CH, Lin BJ, Chuang LM. Mortality trends in patients with diabetes in Taiwan: a nationwide survey in 2000-2009. J Formos Med Assoc. 2012:111:645-50.

32. Umpierrez GE, Isaacs SD, Bazargan N, You X, Thaler LM, Kitabchi AE. Hyperglycemia: an independent marker of in-hospital mortality in patients with undiagnosed diabetes. J Clin Endocrinol Metab. 2002;87:978-82.

33. Thapa R, Joshi DM, Rizyal A, Maharjan N, Joshi RD. Prevalence, risk factors and awareness of diabetic retinopathy among admitted diabetic patients at a tertiary level hospital in Kathmandu. Nepal J Ophthalmol. 2014:6:24-30.

34. Naresh CN, Hayen A, Weening A, Craig JC, Chadban SJ. Day-to-day variability in spot urine albumin-creatinine ratio. Am J Kidney Dis. 2013;62:1095-101.

\section{Submit your next manuscript to BioMed Central and we will help you at every step:}

- We accept pre-submission inquiries

- Our selector tool helps you to find the most relevant journal

- We provide round the clock customer support

- Convenient online submission

- Thorough peer review

- Inclusion in PubMed and all major indexing services

- Maximum visibility for your research

Submit your manuscript at www.biomedcentral.com/submit
O Biomed Central 